\title{
Covid-19, instituciones financieras internacionales y continuidad de las políticas androcéntricas en América Latina
}

\author{
Juan Pablo Bohoslavsky' (iD 0000-0002-7059-281X \\ Mariana Rulli ${ }^{2}$ \\ 'ONU (2014-2020), Ginebra, Suiza. \\ Derechos, Inclusión y Sociedad. Río Negro, Argentina. ciedis@unrn.edu.ar
}

${ }^{2}$ Universidad Nacional de Río Negro. Centro Interdisciplinario de Estudios sobre

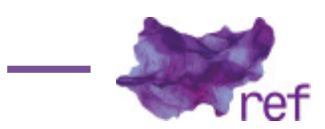

Resumen: Este artículo analiza, desde una perspectiva de derechos humanos y de la economía feminista, las políticas financieras de emergencia desplegadas por las instituciones financieras internacionales (IFls) -sobre todo el FMl y el Banco Mundial- para ayudar a los Estados de América Latina a enfrentar la crisis del Covid-19. Se estudian los presupuestos macroeconómicos y fiscales, así como las políticas económicas subyacentes a los créditos que las IFIs están otorgando a los Estados, identificando claras señales de que la disciplina fiscal y las opciones promercado continuarán siendo las prioridades apenas se supere la emergencia. Se explica de qué modo las políticas de ajuste y la austeridad implementadas en los países de la región han afectado los derechos de las mujeres de manera adversa y desproporcionada, en particular reforzando la invisibilización de las desigualdades de género en el trabajo doméstico y de cuidados, y cómo esa situación, a su vez, ha posicionado a las mujeres en una situación de mayor vulnerabilidad frente a la pandemia. También se critica el (auto)Ilamado "enfoque de género" de las IFls por ser meramente instrumental al crecimiento económico sin consideración por el valor intrínseco de la igualdad de género y los derechos de las mujeres. Se analiza la responsabilidad de las IFls por complicidad con políticas económicas con efectos reconocidamente adversos sobre la igualdad de género. Dadas las implicaciones políticas y legales de los hallazgos que presenta esta investigación, el artículo concluye que los Estados de la región deberían considerar cuidadosamente las condiciones de los préstamos ofrecidos por las IFls, para lo cual resulta esencial realizar evaluaciones ex ante del impacto de tales contratos financieros sobre los derechos humanos de la población, y en particular sobre la igualdad de género.

Palabras claves: Covid-19; desigualdad de género; FMI; Banco Mundial; austeridad; derechos humanos.

Covid-19, Instituições financeiras internacionais e continuidade das políticas androcêntricas na América Latina

Resumo: Este artigo analisa, de uma perspectiva de direitos humanos e da economia feminista, as políticas financeiras de emergência impulsionadas pelas instituições financeiras internacionais (IFIS) notadamente o FMI e o Banco Mundial-para ajudar os Estados da América Latina a enfrentar a crise da Covid-19. Examinam-se os pressupostos macroeconômicos e fiscais, bem como as políticas econômicas subjacentes aos créditos que as IFIs estão outorgando aos Estados, identificando claros sinais de que a disciplina fiscal e as opções pró-mercado continuarão assim que a emergência for superada. O artigo explica de que modo as políticas de ajuste e austeridade implementadas nos países da região afetaram e afetam os direitos das mulheres de forma adversa e desproporcional, em particular reforçando a invisibilização das desigualdades de gênero no trabalho doméstico e de cuidados, e como essa situação, por sua vez, tem posto as mulheres em uma situação de maior vulnerabilidade diante da pandemia. Critica-se também o (auto)chamado "enfoque de gênero" das IFIs por ser meramente instrumental ao crescimento econômico, sem consideração pelo valor intrínseco da igualdade de gênero e dos direitos das mulheres. Analisa-se a responsabilidade das IFIs por cumplicidade com políticas econômicas que geram efeitos reconhecidamente adversos sobre a igualdade de gênero. Dadas as implicações políticas e legais dos achados apresentados por esta pesquisa, o artigo conclui 
que os Estados da região deveriam considerar cuidadosamente as condições dos empréstimos oferecidos pelas IFIs, para o qual é essencial realizar avaliações ex ante do impacto de tais contratos financeiros sobre os direitos humanos da população e, em particular, sobre a igualdade de gênero. Palavras-chave: Covid-19; desigualdade de gênero; FMI; Banco Mundial; austeridade; direitos humanos.

\section{Covid-19, International Financial Institutions and the Continuity of Androcentric Policies in Latin America}

Abstract: This article analyses, from a human rights and feminist economy perspective, the emergency financial policies deployed by the international financial institutions (IFIs) -in particular the IMF and the World Bank- to help States in Latina America to cope with the Covid-19 crisis. The macroeconomic and fiscal assumptions as well as the underlying economic policies behind the loans that IFIs are granting to States are studied, identifying clear signals that fiscal discipline and pro-market options will continue being the priorities as soon as the emergency has been overcome. The study explains how the adjustment and austerity policies implemented in the countries in the region have adversely and disproportionally affected women's rights, in particular reinforcing the invisibilization of gender inequalities in the domestic and care work and how this situation, in turn, has put women in a situation of greater vulnerability to the pandemic. It also criticizes the (self)called "gender approach" of IFls for being merely instrumental to economic growth without consideration to the intrinsic value of gender equality and women's rights. The responsibility of IFIs for complicity with economic reforms with known adverse effects on gender equality is studied. Given the policy and legal implications of the findings presented in this research, the article concludes that States in the region should consider carefully the conditions of the loans being offered by IFIs, for which it is crucial to conduct ex ante evaluations of the impact of these financial contacts on the human rights of the population, and in particular on gender equality.

Key words: Covid-19; Gender inequality; IMF; World Bank; Austerity; Human Rights.

\section{Introducción}

Un gran número de actores y actrices políticos/as abogan -tanto a nivel nacional como internacional- por políticas económicas y sociales para afrontar la crisis sanitaria del Covid-19 y la recesión económica a la que ha sumido al mundo, que coadyuven al fortalecimiento inmediato de los sistemas de protección social y de los sistemas de salud.

Hasta el Fondo Monetario Internacional (FMI), apartándose de sus clásicas recetas promercado y de estricta disciplina fiscal, sostiene que deben implementarse medidas para proteger las vidas de las personas y contribuir a los sustentos de los hogares, incluyendo la asistencia a trabajadores/as, desocupados/as y empresas para restaurar lo antes posible el comercio y los flujos financieros una vez que comience la recuperación económica (Giovanni DELL'ARICCIA, 2020). También el FMl afirma que los hogares que pierdan sus ingresos debido -directa o indirectamentea las medidas de confinamiento necesitarán apoyo gubernamental, y que las prestaciones por desempleo deben ampliarse y extenderse. Aún más explícita ha sido la directora del FMI, Kristalina Georgieva, al señalar el 16 de abril de 2020 que "apoyaremos los esfuerzos de los países para calibrar sus políticas sociales para reducir la desigualdad, proteger a las personas vulnerables y promover el acceso de oportunidades para todos/as" (Kristalina GEORGIEVA, 2020).

Si bien es cierto que en estos documentos y declaraciones no existen referencias explícitas a la discriminación contra las mujeres y niñas, ni a su mayor exposición frente a la crisis sanitaria y económica del Covid-19, uno/a podría otorgar el beneficio de la duda y asumir que cuando la directora del FMI habla de "oportunidades para todos/as" (opportunities for all), ' se está refiriendo también a la (des)igualdad de género. Sin embargo, el diseño y despliegue de los instrumentos financieros y declaraciones políticas del FMl y del Banco Mundial que hemos visto hasta ahora para enfrentar la crisis económica derivada de la pandemia, ¿realmente nos lleva a afirmar que, efectivamente, estamos presenciando un cambio transformador de política de las instituciones financieras internacionales (IFIs), en el que la desigualdad de género es tomada seriamente, 0 , por el contrario, el enfoque económico de las IFIs, que consolida y reproduce las desigualdades de género, se mantiene incólume?

Dado que la implementación de las políticas económicas y sociales promovidas por las IFIs en las últimas décadas han exacerbado la desigualdad de género en numerosos países de América Latina, es necesario y oportuno interrogarnos acerca de la perspectiva de género real de las políticas desplegadas por las IFIs para capear la crisis del Covid-19. La respuesta frente a estos interrogantes debería permear los procesos nacionales de discusión en torno a los beneficios y costos de tomar créditos cada vez más voluminosos, aún a tasas subsidiadas, con

\footnotetext{
' El lenguaje inclusivo "todos/as" fue incorporado por los/as autores/as de este artículo, la versión en inglés se refiere
} a "all". 
condicionalidades que pueden resultar en el mediano y largo plazo demasiado onerosas para los derechos humanos de la población, y en particular para los de las mujeres (Juan Pablo BOHOSLAVSKY, 2018a).

Desde un enfoque de derechos humanos y de los principales lineamientos teóricos de la economía feminista (Marianne FERBER; Julie NELSON, 1993; 2003; Cristina CARRASCO, 2014; Corina RODRÍGUEZ ENRÍQUEZ, 2015), en este artículo abordaremos un interrogante central: las políticas financieras y económicas que promueven las IFls para capear la crisis sanitaria, social y económica del Covid-19, ¿reducen o exacerban las desigualdades de género en América Latina?. Para ello intentaremos desentrañar las políticas económicas subyacentes tanto en el arsenal financiero desplegado por las IFIs en últimos meses para enfrentar la pandemia como en sus documentos oficiales emitidos recientemente (Sección 2). Luego describiremos las formas mediante las cuales los derechos humanos de las mujeres han sido afectados de manera agravada y diferenciada por las políticas de mercantilización de la salud, recortes en el presupuesto estatal y austeridad aplicadas en la última década a instancias de las IFIs en un número países de la región, al mismo tiempo que analizaremos si esas mismas políticas han llevado a que las mujeres sean, a su vez, afectadas de manera desproporcionada por la crisis del Covid-19 (Sección 3.1). También estudiaremos si las (auto)llamadas "políticas de género" promovidas por las IFIs en los últimos años pueden ser consideradas "feministas" (Sección 3.2). Las políticas económicas con reconocidos efectos adversos sobre la igualdad de género no pasan desapercibidas para el derecho internacional de los derechos humanos, con lo que estudiaremos la responsabilidad de las IFIs por promover políticas discriminadoras contra las mujeres (Sección 4). Finalmente presentaremos nuestras conclusiones y las posibles implicaciones legales y de políticas públicas de los hallazgos presentados en este artículo (Sección 5).

El objeto de esta investigación se ha limitado al FMl y Banco Mundial, dados su despliegue inmediato de líneas de crédito masivas en el contexto de la pandemia, que los montos asignados para esas líneas de crédito son inconmensurablemente superiores a los de las demás IFIs, el elevado monto de los créditos ya otorgados a los países de la región antes del brote de la pandemia y, sobre todo, los serios problemas en materia de igualdad de género que han planteado por décadas sus condicionalidades clásicas. La investigación podría ampliarse hacia otros bancos regionales de desarrollo, como el Banco Interamericano de Desarrollo y el Banco de Desarrollo de América Latina, pero sabemos que sus créditos en el actual contexto no son tan voluminosos como los del FMI y el Banco Mundial (NACIONES UNIDAS, 2020c), y que hasta ahora no han sido particularmente intrusivos con condicionalidades de ajuste y austeridad. Por otra parte, aunque hasta ahora sus créditos tampoco han estado públicamente asociados a ese tipo de condicionalidades, dada la presencia financiera china en algunos países de la región -como Ecuador-, será interesante ampliar esta investigación cuando el país asiático defina su estrategia de política financiera hacia los países endeudados, sea a través de nuevos créditos, suspensión, reestructuración o cancelación de los existentes, y/o mediante la participación en el fondo de contención de catástrofes del FMI (Deborah BRAUTIGAM, 2020).

\section{Préstamos y condicionalidades en tiempos de pandemia: La ortodoxia económica de siempre}

En el contexto de la recesión económica provocada por el Covid-19 y las medidas adoptadas para contener los contagios, los gobiernos y la comunidad internacional se comprometen a inyectar billones de dólares en la economía; de hecho, el G20 ha prometido aportar 5 billones de dólares en la economía global y "hacer lo que sea necesario para superar la pandemia" (BBC, 2020). La Unión Europea anunció compromisos "sin límites" para proteger las economías europeas a través de la compra de deuda soberana y corporativa, mientras que el Congreso de Estados Unidos aprobó un paquete de estímulos por USD 2 trillones.

Estas opciones fiscales y financieras no se encuentran disponibles para los países de ingresos bajos y medios que caracterizan a la región latinoamericana. La fuga de capitales (Sergi LANAU; Jonathan FORTUN, 2020), la caída de las exportaciones, de los precios de los commodities y del turismo, la desaceleración económica, la depreciación económica y el agobio del endeudamiento externo conducen a los países en desarrollo, incluidos los de la región, hacia una tormenta perfecta, reduciendo así el margen de maniobra financiera y fiscal de esos países para enfrentar la pandemia. Por ese motivo, las IFIs, con el FMI y el Banco Mundial jugando un rol central en línea con su origen y propósito geopolítico (Benn STEIL, 2016), se han embarcado en programas de préstamos extraordinarios y de alivio de deuda multimillonarios para ayudar a los países en desarrollo a capear la recesión económica.

El FMl está respondiendo a pedidos de créditos de más de 80 países y ha puesto a disposición dos líneas de crédito. En primer lugar, ofrece USD 50.000 millones de rápido desembolso, incluso $1 / 5$ de esos fondos puede otorgarse a tasa nula a países de bajos ingresos y de mercados 
emergentes (FMI, 2020a), pero de todos modos esos préstamos deben contener un programa pleno del FMI (FMI, 2020b). Por otro lado, los países pueden aplicar a los fondos de contención de catástrofes, diseñado para colaborar en contextos de pandemias, por un monto de USD 500 millones. Este programa comenzó a ser ejecutado a principios de abril de 2020 (FMI, 2020c), pero dado su volumen limitado, la mayoría de los países son animados a aplicar a los préstamos convencionales que se encuentran sujetos a las conocidas condicionalidades que deben comenzar a implementarse antes que el dinero sea desembolsado. El FMI también ha anunciado el alivio para el pago de deuda de sus "miembros más pobres" (FMI, 2020d), pero aún no se conocen las condiciones bajo las cuales operará.

El Banco Mundial anunció un paquete de préstamos por USD 14.000 millones, con la esperanza de incrementar varias veces este monto en los próximos 15 meses (BANCO MUNDIAL, 2020). En vez de utilizar su División de Salud, Nutrición y Población, la mayor parte (USD 8.000 millones) de los préstamos se canalizará a través de Corporación Financiera Internacional -el brazo para el financiamiento privado del Banco Mundial- a pesar de su nula experiencia en el desarrollo de sistemas de salud pública y los catastróficos resultados de las asociaciones públicoprivadas en el ámbito de la salud (Arne RUCKERT; Ronald LABONTÉ, 2014). El resto de los fondos está destinado a apoyar el cuidado de la salud en forma directa pero aún no se sabe ciertamente si financiará los sistemas de salud pública o si en cambio financiará clínicas privadas. La segunda opción parece ser la más verosímil, puesto que los préstamos a compañías privadas en el sector de salud están previstos en la línea de créditos disponible (Alexander KENTIKELENIS et al., 2020). Además, el 23 de marzo de 2020, en pleno brote del virus, el presidente del Banco Mundial afirmó que "Ios países necesitarán implementar reformas estructurales para reducir el tiempo de recuperación y crear confianza para que la recuperación pueda ser fuerte. Para aquellos países que tienen regulaciones excesivas, subsidios, regímenes de licencias, protección del comercio y litigiosidad como obstáculos, trabajaremos con ellos para fomentar los mercados, elegir y acelerar las prospectivas durante la recuperación" (David MALPASS, 2020).

En esa línea, el jefe del departamento de asuntos fiscales del FMI señaló, a principios de abril de 2020, a través del llamado "Monitoreo Fiscal" (FMI, 2020e), que una vez que pase la pandemia los países necesitarán adoptar medidas para "poner los indicadores de deuda en una trayectoria descendiente firme", y que es importante que gobiernos "administren las expectativas (...) dejando aclarado que el apoyo a las medidas para afrontar la crisis del Covid-19 son temporarias."

Esas declaraciones hielan la sangre porque sabemos qué significan las reformas estructurales en boca de las IFIs: priorizar ante todo los objetivos fiscales y las soluciones de mercado para afrontar problemas sociales acuciantes, incluidos los desafíos de la salud pública, lo cual se ha traducido regularmente en políticas de consolidación fiscal, ajustes, privatizaciones, desregulación de los mercados financieros y laborales, y relajación de las normas de protección del medio ambiente, todo lo cual tiene probadamente efectos negativos sobre los derechos humanos. Ya lo vimos luego de la crisis financiera global de 2008-9: los países -muchos de la región latinoamericana- que siguieron las recetas económicas ortodoxas vieron reducir su espacio fiscal para asegurar la realización de los derechos humanos, sobre todo los derechos económicos y sociales, y esta política de ajuste golpeó de manera diferenciada a las mujeres (BOHOSLAVSKY, 2018a).

\section{IFIs, austeridad y desigualdades de género}

\subsection{El legado del ajuste y la situación de las mujeres frente a la pandemia}

La discriminación por género en la esfera económica no necesariamente es atribuible a políticas específicas, sino que es sintomática de las estructuras androcéntricas profundamente arraigadas en todas las políticas y teorías económicas dominantes (Paula ENGLAND; Barbara STANEK KILBOURNE, 1990; Shahra RAZAVI, 2007) que, desde una perspectiva latinoamericana, se explica también como el resultado perverso de una matriz más amplia y compleja resultante de la colonialidad del poder (Aníbal QUIJANO; 2002, 2011). Aún ello, el estudio diferenciado de ciertas políticas se encuentra justificado por cuanto mejora nuestra comprensión sobre la relación entre discriminación de género y economía, a la vez que nos interpela para movilizarnos contra las injusticias económicas y sociales.

La austeridad como regla de administración es en sí loable si se la opone al despilfarro, pero quiere decir cosas muy distintas según qué grupos sociales deban ajustar sus ingresos y sus gastos. La impulsada por el FMl y otras IFls no ha sido cualquier austeridad, sino una que no solo no restringe el servicio de la deuda pública que reciben los rentistas locales y extranjeros, sino que provoca el aumento del pago de intereses. Este tipo de políticas monetarias, en cambio, sí suelen reducir subsidios a los alimentos y servicios públicos básicos, los salarios reales, la inversión en 
vivienda e infraestructura, el gasto en investigación, salud y educación, a la vez que descartan invertir fuertemente en una infraestructura social de cuidados robusta. No sorprende comprobar, en los países de la región latinoamericana, que el incremento en el pago de intereses de las deudas redujo los recursos disponibles para el desarrollo. Por ejemplo, entre los años 2010-201 8, mientras que pago de intereses aumentoì del $1,7 \%$ al $2,5 \%$ del PBI, el gasto en salud tuvo un incremento mucho menor (del $1,9 \%$ al 2,3\%) y, a su vez, el gasto de capital se redujo del $3,9 \%$ al $3,2 \%$ del PBI (CEPAL, 2020a).

Los programas de ajuste estructural del FMI y del Banco Mundial han sido criticados por imponer medidas de austeridad que impactaron de manera significativa y desproporcionada en los/as pobres y agravaron la desigualdad, incluida la de género. Esto no es solo una crítica del pasado, puesto que el FMl y el Banco Mundial han continuado prescribiendo políticas que socavan la igualdad de género y los derechos humanos de las mujeres (Grazielle DAVID, 2018). Algunas de estas incluyen privatizar los servicios públicos, mercantilizar la salud (Timon FORSTER et al., 2020), reducir el tamaño de las redes de seguridad social y recortar los salarios, junto con la desregulación laboral, las reducciones en las pensiones, y la profundización de regímenes tributarios regresivos mediante la introducción o el aumento del IVA al tiempo que se reducen las tasas impositivas empresariales (BRETTON WOODS PROJECT, 2017).

Todas estas medidas han situado a los sectores en situación de mayor vulnerabilidad, y especialmente a las mujeres, en una posición desventajosa para afrontar la crisis sanitaria y sus impactos directos e indirectos (NACIONES UNIDAS, 2020b). Más concretamente, el triple peligro de la austeridad condena a las mujeres a sufrir al mismo tiempo como trabajadoras del sector público, como usuarias de los servicios, y como principales beneficiarias de las prestaciones de seguridad social, todo lo cual tiene repercusiones concretas en lo que respecta a seguridad financiera de las mujeres y la distribución de los cuidados. A su vez, ello agrava la discriminación por razón de género en el mercado de trabajo y la segregación ocupacional, y las expone a un mayor riesgo a sufrir contagios del Covid-19, así como violencia de género, incluso la muerte. Veremos en las siguientes secciones que estas desventajas han posicionado a las mujeres en una situación de mayor vulnerabilidad frente a la crisis sanitaria y económica.

\section{La invisibilización y persistencia de las desigualdades en los cuidados}

En todo el mundo, el cuidado de personas remunerado y no remunerado es frecuente y principalmente realizado por mujeres: ellas realizan más de tres cuartas partes del trabajo de cuidados no remunerado y representan las dos terceras partes de la mano de obra que se ocupa del trabajo de cuidados remunerados (Laura ADATTI et al., 2018). En América Latina y el Caribe², las mujeres le dedican diariamente al trabajo doméstico y de cuidados no remunerados el triple del tiempo que le dedican los varones (CEPAL, 2018): en promedio las mujeres realizan el $75 \%$ de estos trabajos. Además, el valor del trabajo no remunerado o pobremente remunerado de las mujeres y su contribución a la economía han sido seriamente subestimados e invisibilizados durante siglos.

Estas desigualdades en los cuidados están cimentadas (y se reproducen) en los estereotipos sociales y los roles asignados a las mujeres vinculados a los cuidados, afectan las trayectorias educativas y las oportunidades laborales de las mujeres que las ubican en posiciones desfavorables para acceder a mejores puestos de trabajo. Desigualdades que se multiplican y agudizan al incluir las múltiples e interrelacionadas formas de discriminación a causa de la condición socioeconómica, edad, raza, orientación sexual o condición de migrante. En Brasil, por ejemplo, según datos del Retrato de Desigualdades de Género y Raza (IPEA), el promedio de años de estudios de las mujeres blancas era para 2015 de 10,6 años, mientras que para las mujeres negras era de 8,9 años. En el caso del mercado de trabajo, la tasa de desocupación de las mujeres blancas era del $9,8 \%$ y de las negras del $13,6 \%$.

Las recomendaciones y condicionalidades impulsadas por las IFls no han sido diseñadas con una perspectiva de género que reconozca la injusta organización social del cuidado en la región latinoamericana. De la mano de los movimientos de mujeres y de la literatura de la economía feminista se fue logrando visibilizar esta desigualdad sistémica y fue incluida en la Plataforma de Acción de Beijing de 1995. Sin embargo, a pesar de que las IFls han captado las demandas feministas e impulsado algunas políticas (autodenominadas) de género (véase la próxima sección), las recomendaciones y condicionalidades vinculadas al mercado de trabajo siguen siendo diseñadas desde una perspectiva ortodoxa que considera al trabajo como aquel que es remunerado, dirigido a producir bienes y servicios y sin reconocer a los trabajos vinculados a la reproducción de la vida.

Probablemente la "política de género" más patriarcal de las IFIs ha sido el desaliento a que los Estados inviertan fuertemente y de manera sustentable en la economía de cuidados. Se han priorizado otros gastos fiscales, como el pago de las deudas a los acreedores externos. No es

${ }^{2}$ Comparativamente, América Latina es una de las regiones más desiguales: en Suecia la contribución de los varones es del $44 \%$, en EE. UU. del $38 \%$ y en China del 39\% (Claudia PIRAS, 2020). 
posible sobreestimar los efectos positivos que aquella inversión tendría sobre los derechos de las mujeres: no solo repercutirían positivamente en los ingresos y tiempo de las mujeres, sino que también podría servir para abordar los factores más determinantes de la desigualdad de género al facilitar un cambio de las normas y valores con perspectiva de género. Las IFIs siquiera han alentado a los Estados a que computen los trabajos de cuidado en sus cuentas nacionales.

Con algunas excepciones, como el Sistema Nacional de Cuidados en Uruguay, en la región latinoamericana se han logrado ciertos avances, pero de manera muy fragmentada (se ha avanzado en las licencias para cuidar, en su mayoría extensiones de la de maternidad) e insuficiente y con políticas dirigidas a las personas que tienen una relación laboral de dependencia o con transferencias de ingresos focalizadas y no de acceso universal. Una señal de la persistente invisibilización de los trabajos de cuidados es que en la mayoría de los países de la región (a excepción de Nicaragua en 1998) recién en 2010 se comenzaron a realizar relevamientos oficiales a través de encuestas de uso del tiempo y en la mayoría de los casos se hizo una sola vez.

Desde marzo de 2020, bajo las recomendaciones de la Organización Mundial de la Salud (OMS) y por la experiencia de los países que sufrieron los embates de la pandemia unas semanas antes, en gran parte de los países de la región latinoamericana se impusieron el distanciamiento social, el aislamiento en los hogares y el cierre de los establecimientos educativos como las principales medidas para evitar y mitigar la propagación del virus. Estas medidas sanitarias agravan la asimetría en los cuidados.

Según UNICEF (2020), al 23 de marzo de 2020 aproximadamente 154 millones de niños, niñas y adolescentes (más del $95 \%$ de los/as que se encontraban matriculados/as) en América Latina se encontraban fuera de las escuelas por la decisión de los gobiernos de cerrarlas temporalmente. ${ }^{3}$ Estas medidas implicaron una sobrecarga de trabajo de cuidados hacia los hogares, de acompañamiento de la educación a distancia, de elevados estándares de limpieza de los hogares y de teletrabajo, por mencionar sólo algunas de las implicaciones. Los Estados deberían disponer de planes de corto y mediano plazo para revertir esa disparidad -aún crecientede responsabilidades.

\section{La precariedad en el trabajo y la protección social. La pobreza como "comorbilidad"}

Las reformas estructurales, sumadas a las políticas de austeridad y desregulación y flexibilización del mercado de trabajo que se implementaron en gran parte de los países latinoamericanos, afectaron los derechos y las condiciones de las mujeres en el ámbito laboral. Debido a la segregación ocupacional (tanto vertical como horizontal), la prevalencia de las mujeres en el sector público y la mayor proporción en los empleos no registrados, el impacto de las medidas gubernamentales para enfrentar la pandemia ha sido asimétrico en términos de género. No es sorprendente que la crisis sanitaria y económica producto de la pandemia golpee más fuerte a las mujeres que a los varones, ya que aquéllas a menudo están sobrerrepresentadas en el sector informal, tienen un trabajo mal pago y mayor probabilidad de perder sus empleos. Según cifras de la Organización Internacional del Trabajo, 126 millones de mujeres trabajan de manera informal en América Latina y el Caribe, aproximadamente la mitad de la población femenina de la región (Karina BATTHYÁNY, 2020). En Bolivia, Guatemala y Perú más del $80 \%$ de las mujeres tienen empleos informales. Las mujeres en América Latina son casi el $40 \%$ de los/as empleados/as de comercio, restaurantes y hoteles, todos sectores altamente afectados por la crisis del coronavirus y que presentan altos grados de informalidad (PIRAS, 2020).

La crisis de los cuidados se manifiesta también en el mercado de trabajo: el 11,4\% de las mujeres ocupadas de la región latinoamericana realizan trabajos domésticos remunerados. Según la OIT (2016), el 77,5\% de las personas del sector realizan sus trabajos en condiciones de informalidad. La vulnerabilidad de las trabajadoras domésticas, en contexto de crisis económica y en la pandemia actual, es mayor debido a la ausencia de regulación en muchos países, las dificultades y menos oportunidades para asociarse y ejercer los derechos laborales y las negociaciones colectivas (quedando así más expuestas frente a los abusos de sus empleadores/as, que en muchos casos las obligan a trabajar aún durante la cuarentena) y, como ya hemos mencionado, la desvalorización e invisibilización de estos tipos de trabajos en la región (CEPAL, 2020b).

Incluso, a pesar de la existencia de regulaciones, como es el caso argentino que desde 2013 tiene una ley especial sobre los/as y trabajadores/as de casas particulares (que incluye diversas categorías entre las que se encuentran los trabajos domésticos y el cuidado de personas), la falta de fiscalización laboral deja a estas trabajadoras en situación de desprotección. En Argentina, el trabajo doméstico se encuentra altamente feminizado, ya que el $98 \%$ de las trabajadoras son mujeres y representa el $16 \%$ de los empleos de las mujeres. A su vez, es la

\footnotetext{
${ }^{3}$ Argentina, Bolivia, Chile, Colombia, el Ecuador, El Salvador, Guatemala, Honduras, Jamaica, Panamá, Paraguay, Perú, Santa Lucia, Trinidad y Tobago, el Uruguay y Venezuela habían suspendido las clases en todos los niveles educativos para el 20 de marzo de 2020. En Brasil se habían suspendido en algunas localidades (CEPAL, 2020a).
} 
actividad con mayor proporción de empleo no registrado: el $75,5 \%$ de las mujeres que realizan trabajos domésticos son trabajadoras informales en condiciones precarias y sin acceso a los beneficios de la protección social (ENCUESTA PERMANENTE DE HOGARES-INDEC 2018). Si no trabajan, no cobran un salario.

En numerosos países, los recortes en los sistemas de protección social han repercutido de muchas maneras en el derecho de las mujeres a la seguridad social, en particular mediante reducciones de diversas prestaciones de desempleo, sociales, familiares o de la ayuda a las personas mayores y a las personas dependientes. Por ejemplo, pocos países en América Latina poseen seguro de desempleo para los/as trabajadores/as formales: en 2019, sólo la Argentina, el Brasil, Chile, Colombia, el Ecuador y el Uruguay contaban con algún tipo de prestación por desempleo (CEPAL, 2020a). Aún si tomamos en cuenta los instrumentos de protección social de emergencia desplegados por los Estados de la región para intentar capear la pandemia, observamos que son esquemas monetarios básicos, de emergencia y no universales, con lo que no abordan realmente los problemas de desigualdad estructurales.

Como ya se ha mencionado, la situación es más grave para las mujeres que sufren formas múltiples e interrelacionadas de discriminación a causa de su condición socioeconómica, edad, raza, orientación sexual o condición de migrante, entre otras dimensiones. Por ejemplo, las familias monomarentales y miembros de grupos de minorías tienen más probabilidades de verse afectadas por los recortes en las prestaciones y las desgravaciones fiscales porque tienen más probabilidades de vivir en la pobreza, con hijos/as a su cargo y familias numerosas, lo que aumenta la carga que soportan esos grupos marginalizados. Es importante tener en cuenta la diversidad de las mujeres, que no pueden considerarse como un grupo monolítico. Las experiencias de las mujeres en diferentes grupos sociales son únicas, y quienes más sufren los efectos de las crisis financieras y sus consecuencias suelen ser quienes menos han contribuido a provocarlas. Habida cuenta de que las mujeres tienen más probabilidades de vivir en la pobreza, las modificaciones y las reducciones de las prestaciones y del gasto público han afectado más gravemente a los grupos más expuestos y marginalizados de mujeres. Ello pone de relieve los efectos acumulativos de los múltiples niveles de discriminación en los derechos humanos de las mujeres.

Las medidas de ajuste y austeridad de los últimos años también tuvieron su impacto en términos de pobreza y han dejado a América Latina en una situación de mayor vulnerabilidad para enfrentar la pandemia: la pobreza viene acompañada por la falta de acceso a viviendas que permitan llevar adelante un aislamiento eficaz, a una atención médica de calidad, a una alimentación saludable, al agua potable para lavarse las manos, a la posibilidad de continuar generando ingresos mediante teletrabajo, y a la educación a distancia. Según la CEPAL (2019), la pobreza aumentó entre 2014 y 2018 en América Latina, la tasa actual de pobreza extrema es de $11 \%$ y la de pobreza del 30,3\%. Dadas las condiciones de desigualdades económicas, sociales y de género en la región, los efectos de la pandemia en el mercado de trabajo como el desempleo, aumento del trabajo no registrado y el incremento en las familias más pobres que envían a sus hijos/as al mercado de trabajo, afectarán desproporcionadamente a los sectores de menores recursos. Cálculos recientes de la CEPAL (2020a) señalan que es probable que, a consecuencia de la caída proyectada del $5,3 \%$ del PBI y el aumento del desempleo en $3,4 \%$, y en consecuencia un efecto negativo directo sobre los ingresos de los hogares y su posibilidad de contar con recursos suficientes para satisfacer las necesidades básicas, la tasa de pobreza aumentaría 4,4\% durante 2020 , al pasar del $30,3 \%$ al $34,7 \%$, lo que significa un incremento de 28,7 millones de personas en situación de pobreza (CEPAL, 2020a). . En América Latina hay mayor proporción de mujeres pobres que varones: en 2018 , el $26,9 \%$ de las mujeres eran pobres, y el $23,8 \%$ de los varones. En el caso de la pobreza extrema, el $11,4 \%$ de las mujeres lo eran, y el 9,7\% de los varones (CEPAL, 2019). En el contexto de la pandemia, en los hogares de menores ingresos las desigualdades de género se exacerban, por ejemplo, porque las demandas de cuidados son mayores al contar con mayores personas dependientes (CEPAL, 2020b).

Asimismo, las medidas de austeridad en las últimas décadas han provocado que la desigualdad en el acceso a los servicios básicos sea una problemática grave en la región con impacto desigual en términos de género: en 2018, el 13,5\% de los hogares de la región no tenía acceso a fuentes de agua mejoradas y el $25,4 \%$ en zonas rurales. Las mujeres que habitan en hogares con privaciones de agua potable le dedican al trabajo doméstico y de cuidados no remunerado entre 5 y 12 horas más que las que habitan en hogares con acceso a estos servicios (CEPAL, 2020b). En ese contexto, las mujeres no pueden acceder plenamente al servicio de provisión de agua continua y de calidad para tomar medidas de higiene preventiva frente al coronavirus.

\section{Cuidar en casa y también en el hospital}

En la mayoría de los países de América Latina los sistemas nacionales de salud fueron reformados bajo la influencia del Consenso de Washington. Estas reformas estructurales implicaron una liberalización del sector de la salud, el incremento de su mercantilización y la retracción de 
los sistemas públicos sanitarios. Consecuentemente, los sistemas de salud en América Latina se caracterizan por su segmentación y fragmentación que genera profundas desigualdades en el acceso universal y en la calidad de los servicios de salud. Además, los sistemas de salud suelen estar geográficamente centralizados con los servicios médicos concentrados en los grandes centros urbanos (CEPAL, 2020a).

Las recientes medidas de austeridad implementadas en la región han socavado la capacidad de respuesta de los sistemas de salud. En Argentina, durante el gobierno de Macri el Ministerio de Salud fue reducido a Secretaría con las implicaciones institucionales, de autonomía y presupuestarias que ello implica. ${ }^{4}$ En Ecuador, por ejemplo, un préstamo reciente del FMI que incluía reducciones en el empleo público y en los salarios, implicó el despido de 2.500 trabajadores/ as de la salud (María José ROMERO et al., 2020), en su mayoría mujeres.

Asimismo, en los últimos años, la mayoría de los países de América Latina no han invertido lo suficiente en salud: en 2018, el gasto público de los gobiernos centrales en el sector se situaba en un 2,2\% del PBI regional (CEPAL, 2019), por debajo del $6 \%$ del PBI que recomienda la Organización Panamericana de la Salud para reducir las inequidades y aumentar la protección financiera en el marco del acceso y la cobertura universal (CEPAL, 2020a). En Brasil, a causa de la reforma constitucional-95 de 2016, que impuso el congelamiento del gasto público por 20 años, se ha registrado en los últimos años un recorte continuo en el presupuesto de salud que explica que el Sistema Unificado de Salud cuente solo con la mitad de las camas hospitalarias recomendadas por la Organización Mundial de la Salud.

Los recortes de los presupuestos de salud impactaron tanto en los varones como en las mujeres. Sin embargo, los efectos negativos se han repartido de forma desproporcionada, debido a que las mujeres utilizan más los servicios de atención de salud en gran parte a necesidades propias de su género y a la desventaja inducida del sistema económico (Kate DONALD; Nicholas LUSIANI, 2017). Las medidas de austeridad afectan, específicamente, a la salud reproductiva de las mujeres. Los ajustes presupuestarios provocaron la reducción en los suministros de anticonceptivos de acceso gratuito y, consecuentemente, incrementan los embarazos no deseados, la tasa de mortalidad materna y abortos en condiciones riesgosas. A su vez, la experiencia de brotes anteriores ha indicado que el desvío de fondos y recursos de salud a atender la pandemia incrementan las dificultades para garantizar la atención en la salud prenatal y posnatal, así como el acceso a anticonceptivos (Sophie HARMAN, 2015).

Los recortes del gasto que influyen en el nivel de servicio prestado o el tiempo de hospitalización pueden tener grandes repercusiones para los cuidadores/as y el trabajo de cuidados en su conjunto. En el contexto actual, el cuidado de las personas enfermas puede recaer a menudo sobre los familiares, que probablemente serán mujeres. En México, por ejemplo, se calculó que el valor monetario de los cuidados de salud realizados en el hogar equivalía al $85 \%$ del valor de los servicios que se realizan en los hospitales. Las mujeres aportaban con su trabajo en un $72,2 \%$ de ese valor monetario (CEPAL, 2017).

La presión de la pandemia del COVID-19 sobre los sistemas de salud expone de manera desigual a las mujeres, ya que éstas componen el $72 \%$ del total de las personas ocupadas en ese sector en América Latina (CEPAL 2020b), lo que representa el porcentaje más alto del mundo. Según la Organización Mundial de la Salud, en América Latina el $86 \%$ de los/as enfermeros/as son mujeres y en promedio las trabajadoras del sector ganan un $25 \%$ menos que los varones (CEPAL, 2019). A ello se debe agregar el papel que desempeñan las trabajadoras de la salud comunitarias que no reciben remuneración alguna, las trabajadoras en las farmacias y centros de atención de salud.

Los/as trabajadores/as de los sistemas de salud, en su mayoría mujeres, están trabajando en condiciones extremas de presión y agotamiento que incluye largas jornadas laborales, riesgo de contagiarse por el virus, estrés por las situaciones de emergencia y angustia. Y, a su vez, estas mujeres regresan a sus hogares en los que las espera la sobrecarga de trabajo no remunerado vinculado a las personas dependientes o que requieren de cuidados en sus hogares. Otra vez, esta situación se explica, en gran medida, por la falta de perspectiva de género en las políticas económicas que han promovido las IFIs, cuyas condicionalidades no dejan lugar a una inversión seria en la economía de cuidados, que, según el Women's Budget Group, debería ser del orden del $2 \%$ del PBI.

\section{Ajuste, pandemia y violencias}

La violencia que sufren las mujeres y las niñas en sus diversas formas es tanto una consecuencia como un factor que contribuye a la perpetuación del estigma y los estereotipos al tiempo que refuerzan la explotación económica de forma cíclica, que podría traducirse en menos poder decisorio, acceso a la educación y control de los recursos, lo cual limita sus oportunidades y autonomía. Las medidas de austeridad y la consolidación fiscal dan lugar al debilitamiento de la respuesta estatal frente a la violencia, con consecuencias importantes en el acceso a la justicia. En Brasil, por ejemplo,

${ }^{4}$ El nuevo gobierno que asumió en diciembre de 2019, en una de sus principales medidas, reconstituyó nuevamente al Ministerio de Salud. 
a partir de la Enmienda Constitucional Nro. 95, no se han construido nuevos refugios para mujeres desde 2017 , mientras que ese país tiene actualmente una de las mayores tasas de feminicidio del mundo y ha experimentado recientemente un aumento de la violencia contra las mujeres. En Argentina, en los últimos años, se ha producido un alto porcentaje de subejecución del presupuesto en los programas contra la violencia de género a pesar de que los casos de femicidios son alarmantes. Es oportuno señalar que la subejecución presupuestaria ha sido identificada como una técnica encubierta de implementación de políticas de consolidación fiscal.

En contexto de emergencia sanitaria y medidas de aislamiento social aumentan los riesgos de violencia contra mujeres y niñas. Debido al aumento de trabajo doméstico y de cuidados que ha recaído sobre los hogares, las tensiones económicas y los efectos psicológicos del encierro provocan aumento de tensiones en el hogar y llevan a un incremento en la violencia doméstica. Además, tal como lo señala ONU Mujeres (2020), las personas sobrevivientes de violencia pueden enfrentar obstáculos adicionales, como restricciones de la circulación y acceso a centros de salud, así como para acceder al sistema de protección y/o servicios esenciales. El impacto económico negativo de la crisis sanitaria también puede incrementar las barreras económicas ya existentes y las posibilidades para dejar a una pareja violenta.

Sólo en Argentina, desde el 20 de marzo al 16 de abril de 2020 se cometieron 21 femicidios de mujeres y niñas. En el mismo período del año anterior hubo 14 femicidios. Es decir, en contexto de aislamiento hubo un $50 \%$ más de femicidios respecto del año anterior. Una de cada 5 de esas mujeres habían realizado denuncias previas y el $65 \%$ fueron asesinadas en sus hogares en contexto de aislamiento. ${ }^{5}$ Desde el inicio de la cuarentena en marzo de 2020, las llamadas de emergencia por violencia doméstica han aumentado un $25 \%$ (ONU, 2020).

El riesgo de que esta crisis repercuta sumando aún más trabajo de cuidado para las mujeres y exponiéndolas a grados más altos de discriminación y violencia de género, y a sus formas múltiples y cruzadas (RODRÍGUEZ ENRÍQUEZ; PAUTASI, 2016), es tan real como agudo. Es importante, entonces, que el tiempo y los derechos de las mujeres no se conviertan, una vez más y como ha sucedido históricamente, en el factor de ajuste del que los Estados disponen para afrontar la crisis sanitaria y los futuros escenarios económicos bajo las recomendaciones y condicionalidades, una vez más, de las IFIs.

\section{Las IFIs sostienen que ya son feministas, ¿lo son?}

Ya en los noventa había comenzado en América Latina un proceso gradual de apropiación política y cultural de la categoría de género en manos de las políticas neoliberales auspiciadas e implementadas en la región, que llevó a gobiernos -e incluso un gran número de organizaciones de la sociedad civil- a identificar la "perspectiva de género" con modernidad y avanzar hacia un supuesto desarrollo tecnocrático en las políticas públicas, pero a costo de sanear el radicalismo político de los feminismos (Sonia ÁLVAREZ, 1998).

Pero fue los últimos años que el FMI ha promovido abiertamente los "presupuestos con perspectiva de género" como medio eficaz para utilizar las políticas fiscales para combatir las desigualdades entre los géneros. Parte de esta labor ha servido de base para el asesoramiento normativo y la asistencia técnica en países como Argentina, Austria, Bahréin e Irán (FMI, 2018). Sin embargo, el enfoque que el FMl y varios Estados miembros han adoptado recientemente para abordar la desigualdad de género parece ser esencialmente instrumental, es decir, como medio para lograr los objetivos de crecimiento económico. De manera correlativa, y desde esa misma perspectiva instrumental, si la desigualdad de género facilita el crecimiento económico no sería algo malo después de todo.

La igualdad de género puede justificarse por motivos intrínsecos, como objetivo en sii mismo, en relación con argumentos basados en la realización efectiva de los derechos humanos. Sin embargo, la igualdad de género también ha intentado justificarse por razones instrumentales, es decir, como medio para lograr los objetivos de crecimiento económico. Este enfoque instrumental entra en serio conflicto con la importancia intrínseca de la igualdad de género como un componente clave de los derechos humanos, en particular a la luz de las políticas económicas propuestas y promovidas por las IFIs en los últimos años. Si bien algunas investigaciones muestran que garantizar ciertos derechos humanos es bueno para el crecimiento y para la distribución de sus beneficios (Sigrid KOOB; Stinne JORGENSEN; Hans-Otto Sano SANO, 2018), no hay evidencia concluyente que demuestre que la igualdad de género siempre sea buena para el crecimiento. De hecho, otra evidencia demuestra que la desigualdad de género puede conducir a algunas formas de crecimiento económico (Alison VÁsCONEZ RODRÍGUEZ, 2017).

$\mathrm{Si}$ los gobiernos y las IFIs velan realmente por que los beneficios del crecimiento se distribuyan equitativamente, las justificaciones instrumentales podrían complementar los argumentos intrínsecos basados en los derechos humanos. Esa distribución podría lograrse con una serie de

\footnotetext{
${ }_{5}^{5}$ Casa del Encuentro. Disponible en http://www.lacasadelencuentro.org/index.html, consultado el 21 de abril de 2020.
} 
medidas, como la oferta de incentivos financieros, los servicios sociales, la tecnología y la infraestructura, la recaudación de ingresos suficientes y la creación de oportunidades económicas para todos/as.

El fracaso del FMl en el abordaje de las barreras estructurales que impiden el pleno goce de los derechos económicos y sociales de las mujeres, como la violencia contra las mujeres y las niñas, y su silencio continuado sobre la falta de una inversión pública robusta y sustentable en la economía del cuidado, los impactos de los flujos financieros ilícitos, los regímenes de impuestos regresivos y la privatización de los servicios públicos que afectan los derechos humanos de las mujeres, son también un reflejo de los puntos ciegos del FMl cuando se trata de intervenciones a través de políticas de género justas y eficaces.

Mientras tanto, el "trabajo de género" del FMl sigue enfocándose, en gran medida, en los efectos positivos que cerrar las brechas de género en la participación de la fuerza laboral puede tener sobre el crecimiento. Si bien podría darse el caso de que una políica específica que aliente a las mujeres a ingresar en la fuerza laboral remunerada sea buena para el crecimiento, si ese ingreso no se produce en igualdad de condiciones con los hombres y no se presta atención a los estándares de "trabajo digno" acordados internacionalmente, ese acceso podría reforzar la desigualdad de género al construir una economía en torno a desigualdades estructurales integradas en los mercados laborales.

De manera similar, si bien la Estrategia de Género 2016-2023 del Banco Mundial (BANCO MUNDIAL, 2017) tiene en cuenta algunos obstáculos para la participación económica de las mujeres, algunos/as sostienen (BRETTON WOODS PROJECT, 2016) que se necesitaría una comprensión más amplia del empoderamiento económico de las mujeres en áreas relacionadas con el trabajo para lograr una igualdad sustantiva y que el Banco continúa presionando por las mismas políticas macroeconómicas que el FMI. El Informe sobre el Desarrollo Mundial (BANCO MUNDIAL, 2019) sobre la naturaleza cambiante del trabajo constituye la prueba más reciente de la agenda pronegocio y orientada al crecimiento que infravalora la importancia los derechos laborales y la igualdad de género.

Los programas de ajuste estructural del FMl y del Banco Mundial han suscitado críticas por imponer duras medidas de austeridad que han repercutido de manera significativa y desproporcionada en los/as pobres y exacerbado las desigualdades, incluida la desigualdad de género. Todavía en 2019 el FMI prescribía políticas que socavan la igualdad de género y la realización de los derechos humanos de las mujeres. Entre esas prescripciones se encuentran las siguientes: reducción de los subsidios de los alimentos, privatización de los servicios públicos, reducción de las redes de seguridad social y los salarios públicos, junto con desregulación laboral, disminuciones de las pensiones, recortes de los servicios públicos y regímenes fiscales regresivos, mediante la introducción o aumento del IVA, junto con una reducción de las tasas del impuesto sobre las sociedades (BRETTON WOODS PROJECT, 2017).

\section{Responsabilidad de las IFls por promover políticas discriminatorias contra las mujeres}

Las medidas de austeridad tienen normalmente una serie de efectos probadamente negativos en los derechos humanos, incluida la prohibición de discriminar por razón de género (BOHOSLAVSKY, 2019); por lo tanto, existe una sólida base jurídica para defender la incompatibilidad prima facie entre las políticas de austeridad durante las épocas de recesión y la obligación de proteger el goce de los derechos humanos (BOHOSLAVSKY, 2018b).

No existen pruebas de que siquiera exista la llamada "austeridad expansionista" desde el punto de vista económico (Robert SKIDELSKY; Nicolo FRACCAROLI, 2017). Está mucho más clara la relación entre los programas de ajuste estructural y la reducción del crecimiento económico, el empleo, la sostenibilidad de la deuda y la igualdad. No sorprende que la combinación de desaceleración económica y cambios contractivos de la política fiscal hayan afectado a una amplia variedad de derechos humanos, en particular los derechos de las mujeres (BOHOSLAVSKY, 2018 a). Es evidente que la amenaza de recorte del gasto público cuando y donde más se necesita entraña un elevado riesgo de violación de los derechos humanos.

No es que todas las políticas de reforma que responden a crisis económicas sean intrínsecamente contrarias a los derechos humanos, pero la austeridad carece de una justificación teórica y empírica robusta desde la perspectiva de los derechos humanos. Con arreglo a las normas del derecho internacional, puede considerarse a las IFIs responsables de complicidad en las reformas económicas que violan los derechos humanos, entre ellos el principio de igualdad de género. El vínculo causal entre la asistencia prestada (préstamos, vigilancia y asistencia técnica, y condicionalidades asociadas) en la comisión de un hecho ilícito (complicidad) y el daño causado (violaciones de los derechos humanos) es evidente y está bien documentado. Se puede presuponer el conocimiento del carácter ilícito del acto si, incluso al impulsar reformas económicas 
que normalmente dan lugar a violaciones de los derechos humanos, no se lleva a cabo una evaluación ex ante del impacto. La responsabilidad jurídica por complicidad acarrea obligaciones en términos de cese, no repetición y reparación (BOHOSLAVSKY, 2019).

Debido a las circunstancias en que suelen encontrarse los Estados cuando solicitan asistencia a las IFIs, a menudo se imponen condicionalidades que no se negocian necesariamente con los Estados prestatarios, por no mencionar a su población, a la que se involucra aún menos en las consultas, debates y negociaciones conexas. Además, el alcance de las condicionalidades se ha ampliado continuamente en las últimas décadas (KENTIKELENIS et al., 2016). Todo esto ayuda a entender la generalización y la omnipresencia de las condicionalidades en asuntos soberanos fundamentales, incluso teniendo en cuenta el rechazo abrumador de las respectivas poblaciones y los objetivos de orientación social de las IFls con arreglo a sus propios estatutos.

El hecho de que las IFIs (y los Estados) no realicen ni soliciten normalmente evaluaciones del impacto en los derechos humanos (incluido el de la igualdad de género) es incongruente con su práctica, aunque sea muy imperfecta, de llevar a cabo evaluaciones de los efectos sociales y ambientales cuando se trata de la financiación de proyectos. Si se puede responsabilizar a las IFls por el daño evitable causado a los afectados por una represa financiada, ¿por qué no deberían ser responsables por la violación evitable provocada al principio de igualdad de género por reformas económicas regresivas?

\section{Conclusiones e implicaciones legales y de políticas públicas}

La mayor inversión estatal que demandan las medidas gubernamentales para enfrentar la pandemia y sus efectos económicos y sociales están siendo financiadas por los Estados con mayor déficit fiscal y emisión monetaria, en algunos casos movilizando recursos domésticos limitados a través de políticas tributarias más progresivas, y endeudamiento soberano. Las IFIs están facilitando programas de alivio de deuda y líneas masivas de crédito de emergencia, que en muchos casos están llevando, presumiblemente, a situaciones de sobreendeudamiento soberano y así a una mayor vulnerabilidad frente a las futuras presiones de los acreedores multilaterales (Douglas REDIKER; Heidi CREBO-REDIKER, 2020).

Las condicionalidades y presupuestos ideológicos con las que normalmente están asociados las políticas financieras de las IFIs (Julio GAMBINA, 2020) no parecen apartarse de la ortodoxia y androcentrismo económico que ha caracterizado a esas instituciones en las últimas décadas: priorizar objetivos fiscales de corto plazo a raja tabla, creciente mercantilización de un número de servicios públicos y desregulación. El FMI no deja lugar a dudas acerca de la continuidad de sus políticas macroeconómicas: "Una vez que se haya superado la crisis del Covid-19, los países con deudas altas deberán, en general, procurar una consolidación fiscal apoyada por medidas pro crecimiento" (FMI, 2020e).

La exigencia de implementación de estas políticas económicas con probados efectos regresivos sobre los derechos humanos y la igualdad de género constituye una posibilidad, una amenaza real. No se conocen declaraciones de funcionarios/as ni documentos de las IFls que, por ejemplo, destaquen la importancia mayúscula e inmediata en actual contexto de invertir fuertemente en la economía de cuidados como un acelerador de un cambio estructural con igualdad. La instrumentalización de la igualdad de género y las políticas económicas patriarcales -y la ideología que las motoriza- de las IFIs no parece suspenderse ni aún durante la pandemia del Covid-19. Si bien pareciera que las IFls perciben la necesidad de dar respuestas para fortalecer de manera inmediata los sistemas de protección social para evitar catástrofes humanitarias, no existe en esa percepción una perspectiva de género sensible a la situación diferenciada, agravada de las mujeres durante la pandemia. Planes de cobertura de salud universal o sistemas de protección social universales permanentes tampoco forman parte del menú de políticas de las IFIs.

El sistema económico actual se sustenta en la desigualdad de género y múltiples formas de discriminación. Sin embargo, las IFIs desatienden las formas en que la mayor parte de sus recetas de políticas macroeconómicas han socavado aún más los derechos de las mujeres (Gunseli BERIK, 2017), lo que a su vez explica por qué ahora, nuevamente, las mujeres están más expuestas frente a los efectos de la pandemia y la recesión económica. La invisibilización -histórica y sistémica- del trabajo doméstico y de cuidados no remunerados sumado a las políticas de austeridad que, a través de los recortes en los sectores de la salud, educación y protección social, sumado a la mercantilización y privatización del sector de salud, y la desregulación del mercado laboral que profundiza la segregación ocupacional tanto vertical como horizontal, han afectado a las mujeres de una manera desproporcionada. Todo ello las ha sumido en un lugar aún más expuesto frente al Covid-19 y la crisis que éste provoca. La crisis sanitaria ha puesto en evidencia y demostrado dramáticamente lo que gran parte del feminismo ha venido considerando como fundamental para repensar una organización social del cuidado (RAZAVI, 2007) igualitaria y que ponga en el centro a la sostenibilidad de la vida (CARRASCO, 2014): que todos y todas somos interdependientes (BATTHYÁNY, 2020). 
Las IFI y otros acreedores deberían volcar sus recursos financieros para ayudar a los países a combatir la pandemia (BOHOSLAVSKY, 2020), y garantizar que la aprobación de cualquier préstamo o donación no dependa de la implementación, presente o futura, de condicionalidades como medidas de austeridad, privatizaciones o mercantilización de bienes o servicios esenciales (CEPAL, 2020; Rick ROWDEN, 2019), o ajustes estructurales, con el riesgo de impactar negativamente en los derechos humanos (NACIONES UNIDAS, 2020), en particular en los de las mujeres. En todo caso, los préstamos o cancelaciones de deuda podrían estar vinculadas al aumento sustancial del gasto interno en protección social con el énfasis puesto en salud, educación, nutrición y cuidados, por ejemplo. Esta sería una gran oportunidad para que las IFIs operen según los principios de los derechos humanos en línea con la Carta de la ONU, que deben respetar.

El Consejo de Derechos Humanos de la ONU en su sesión de marzo 2019 votó los Principios Rectores relativos a las evaluaciones de los efectos de las reformas económicas en los derechos humanos (BOHOSLAVSKY, 2018b). Tales Principios Rectores establecen que las evaluaciones de los efectos en los derechos humanos deben ser un elemento central de todos los programas de reforma y ajuste económico, previniendo o minimizando así las violaciones de los derechos humanos. Ello se aplica también a los programas preparados con IFIs, prestamistas bilaterales y donantes públicos en el contexto de la gestión de la deuda y las actividades de asistencia financiera (Principio 15.1). Del mismo modo, las evaluaciones de los efectos en los derechos humanos deberían incluir siempre un análisis de género exhaustivo (Principio 8). No se conoce hasta ahora que este ejercicio de evaluación ex ante se haya llevado a cabo en el contexto de la asistencia multimillonaria que las IFls están desembolsando para paliar los efectos de la crisis del Covid-19 a fin de determinar cómo esos acuerdos financieros y sus condiciones impactarán sobre los derechos de las mujeres.

Tal evaluación de impacto se encontraría en línea con lo que Comisión Interamericana de Derechos Humanos recomendó a los Estados de la región en abril de 2020 (CIDH, 2020): asegurar que las instituciones multilaterales de financiamiento e inversión implementen garantías para proteger los derechos humanos en sus procesos de evaluación de riesgo y sistemas de operación relativos a proyectos de inversión o préstamos monetarios que se den en el contexto de respuesta a la pandemia.

La combinación de la recesión económica que ya golpea a los países de América Latina, el aumento de la deuda multilateral y la continuidad de las políticas ortodoxas (androcéntricas) de las IFIs no parece ser una noticia auguriosa para las mujeres de la región.

\section{Agradecimiento}

Los/as autores/as agradecen a Grazielle David por sus comentarios y sugerencias a borradores de este artículo, y a Celina Lagrutta por su colaboración con cuestiones idiomáticas.

\section{Referencias}

ADATTI, Laura et al.. Care work and Care jobs for the future of decent work. Geneva: ILO, 2018. Disponible en: https://www.ilo.org/global/publications/books/WCMS_633135/lang—en/index.htm

ÁLVAREZ, Sonia, "Feminismos Latinoamericanos". Revista Estudos Feministas, Florianópolis, v. 6, n. 2, p. 265,1998 . doi: https://doi.org/10.1590/\%x

BANCO MUNDIAL, "World Bank Group gender strategy (FY16-23): gender equality, poverty reduction and inclusive growth", 2017, WDC. Disponible en http://documents.worldbank.org/curated/en/ $820851467992505410 / W o r l d-B a n k-G r o u p-g e n d e r-s t r a t e g y-F Y 16-23-g e n d e r-e q u a l i t y-p o v e r t y-$ reduction-and-inclusive-growth

BANCO MUNDIAL, "World Bank Group launches first operations for COVID-19 (coronavirus) emergency health support, strengthening developing country responses", 2 de abril de 2020. Disponible en https://www.worldbank.org/en/news/press-release/2020/04/02/world-bank-group-launches-firstoperations-for-covid-19-coronavirus-emergency-health-support-strengthening-developing-countryresponses

BANCO MUNDIAL. "World Development Report 2019. The Changing Nature of Work", 2019, WDC. Disponible en https://www.worldbank.org/en/publication/wdr2019

BATTHYÁNY, Karina. "La pandemia evidencia y potencia la crisis de los cuidados". Observatorio Social del Coronavirus de CLACSO, Buenos Aires, 2020. Disponible en http://biblioteca.clacso.edu.ar/ clacso/se/2020051 1063748/1-Karina-Batthyany.pdf

BBC. "Coronavirus: Asia shares up on multi-trillion dollar stimulus hopes", 27 de marzo de 2020. Disponible en https://www.bbc.com/news/business-52058984 
BERIK, Gunseli. "Beyond the rhetoric of gender equality at the World Bank and the IMF". Canadian Journal of Development Studies, v. 38 n. 4, p. 564-569, 2017.

BOHOSLAVSKY, Juan Pablo. "Covid-19: Llamamiento urgente para una respuesta a la recesioìn econoimica desde los derechos humanos", 15 de abril de 2020, OHCHR. Disponible en https://www. ohchr.org/Documents/Issues/Development/IEDebt/20200414_IEDebt_urgent_appeal_COVID19_sp.pdf

BOHOSLAVSKY, Juan Pablo. "Informe sobre responsabilidad por complicidad de las instituciones financieras internacionales en las violaciones de los derechos humanos en el contexto de las reformas econoìmicas regresivas". In ASAMBLEA GENERAL DE LAS NACIONES UNIDAS, A/74/1 78, p. 325, 16 de julio de 2019. Disponible en https://undocs.org/pdf?symbol=es/A/74/178.

BOHOSLAVSKY, Juan Pablo. "Impacto de las reformas econoìmicas y medidas de austeridad en los derechos humanos de las mujeres". In ASAMBLEA GENERAL DE LAS NACIONES UNIDAS, A/73/179, p. 1-8, 18 de julio de 2018a. Disponible en https://www.cwgl.rutgers.edu/docman/reports/834-el-impactode-las-reformas-economicas-y-medidas-de-austeridad-en-los-derechos-humanos-de-las-mujeres/file

BOHOSLAVSKY, Juan Pablo. "Principios rectores relativos a las evaluaciones de los efectos de las reformas econoìmicas en los derechos humanos". In ASAMBLEA GENERAL DE LAS NACIONES UNIDAS, $\mathrm{A} / \mathrm{HRC} / 40 / 57,19$ de diciembre de 2018b.

BRAUTIGAM, Deborah. "Chinese Debt Relief: Fact and Fiction". The Diplomat, 15 de abril de 2020. Disponible en https://thediplomat.com/2020/04/chinese-debt-relief-fact-and-fiction/

BRETTON WOODS PROJECT. "El Banco Mundial publica su nueva estrategia de género". Observer, Bretton Woods Project, 16 de abril de 2016, https://www.brettonwoodsproject.org/es/2016/04/elbanco-mundial-publica-su-nueva-estrategia-de-genero/

BRETTON WOODS PROJECT. The IMF and Gender Equality: A Compendium of Feminist Macroeconomic Critiques. London: Bretton Woods Project, octubre de 2017. Disponible en https:// www. brettonwoodsproject.org/wp-content/uploads/2017/10/The-IMF-and-Gender-Equality-ACompendium-of-Feminist-Macroeconomic-Critiques.pdf

CARRASCO, Cristina. "La economiìa feminista: ruptura teoìrica y propuesta poliitica". En Cristina Carrasco (Ed.). Con voz propia. La economiìa feminista como apuesta teoìrica y poliitica. Barcelona: La Oveja Roja, 2014. p. 25-48.

CEPAL. "Dimensionar los efectos del COVID-19 para pensar en la reactivacioìn", Informe Especial No 2, abril de 2020. Disponible en https://repositorio.cepal.org/bitstream/handle/1 1362/45445/1/ S2000286_es.pdf

CEPAL. "La pandemia del COVID-19 profundiza la crisis de los cuidados en América Latina y el Caribe", 2 de abril de 2020b. Disponible en https://repositorio.cepal.org/handle/1 1362/45335

CEPAL. "Panorama Social de América Latina”. LC/PUB.2019/22-P/Rev.1, Santiago, 2019.

CEPAL. "Los cuidados en América Latina y el Caribe: textos seleccionados 2007-2018". Páginas selectas de la CEPAL, LC/M.2018/4, Santiago, 2018.

CEPAL. "Panorama Social de América Latina". LC/PUB.2017/12-P, Santiago, 2017.

COMISIÓN INTERAMERICANA DE DERECHOS HUMANOS. "Pandemia y Derechos Humanos en las Ameìricas", Resolución 1/2020, 10 de abril de 2020. Disponible en http://www.oas.org/es/cidh/ decisiones/pdf/Resolucion-1-20-es.pdf

DAVID, Grazielle. "Los impactos de la austeridad apoyada por el FMI sobre los derechos de las mujeres en Brasil”. El Observador, Bretton Woods Project, 2 de mayo de 2018.

DELL'ARICCIA, Giovanni. "Políticas económicas para la guerra contra el COVID-19". IMF Blog, 1 de abril de 2020. Disponible en https://blog-dialogoafondo.imf.org/?p=13126

DONALD, Kate; LUSIANI, Nicholas. "The gendered costs of austerity: assessing the IMF's role in budget cuts which threaten women's rights". Septiembre de 2017. Disponible en www.brettonwoodsproject.org/ wp-content/uploads/2017/09/The-IMF-Gender-Equality-and-Exp enditure-Policy-CESR-and-BWP-Sept2017.pdf

ENGLAND, Paula; STANEK KILBOURNE, Barbara. "Feminist Critiques of the Separative Model of Self: Implications for Rational Choice Theory". Rationality and Society, v. 2, n. 2, p. 156, 1990. 
ENCUESTA PERMANENTE DE HOGARES- Instituto Nacional de Estadísticas y Censo, Argentina, 2018.

FERBER, Marianne; NELSON, Julie (Eds.). Beyond Economic Man. Chicago: The University of Chicago Press, 1993.

FERBER, Marianne; NELSON, Julie. Feminist Economics Today: Beyond Economic Man, ChicagoLondres: The University of Chicago Press, 2003.

FMI - FUNDO MONETARIO INTERNATIONAL. "How to operationalize gender issues in country work". Documento de politica 2018. Disponible en http://www.imf.org/en/Publications/Policy-Papers/lssues/ 2018/06/13/pp060118howto-note-on-g ender

FMI. "EI FMI ofrece USD 50.000 millones para hacer frente al coronavirus". 4 de marzo de 2020a. Disponible en https://www.imf.org/es/News/Articles/2020/03/04/sp030420-imf-makes-available-50billion-to-help-address-coronavirus

FMI. "Questions and answers on the IMF's $\$ 50$ billion rapid-disbursing emergency financing facilities". 19 de marzo de 2020b. Disponible en https://www.imf.org/en/About/FAQ/50-billion-rapid-disbursingemergency-financing-facilities

FMI. "IMF Executive Board Approves Immediate Debt Relief for 25 Countries". 13 de abril de 2020c. Disponible en https://www.imf.org/en/News/Articles/2020/04/13/pr20151-imf-executive-boardapproves-immediate-debt-relief-for-25-countries

FMI. "El FMI incorpora mejoras al Fondo Fiduciario para el alivio de la Deuda a fin de brindar apoyo a los países de bajo ingreso habilitados tras la pandemia de COVID-19". 27 de marzo de 2020d. Disponible en https://www.imf.org/es/News/Articles/2020/03/27/pr201 16-imf-enhances-debtrelief-trust-to-enable-support-for-eligible-lic-in-wake-of-covid-19; ver también https://www.imf.org/ es/News/Articles/2020/03/27/pr20114-joint-statement-by-the-chair-of-imfc-and-the-managingdirector-of-the-imf

FMI. "Fiscal Monitor Report”. abril de 2020e. WDC, https://www.imf.org/en/Publications/FM/Issues/ 2020/04/06/fiscal-monitor-april-2020

FORSTER, Timon et al. " Globalization and health equity: The impact of structural adjustment programs on developing countries". Social Science \& Medicine, 2020 (en prensa).

GAMBINA, Julio, "La pandemia del Covid-19 agrava la crisis capitalista". Colección Pensar la Pandemia, n. 27, CLACSO, Buenos Aires, 2020c. Disponible en http://biblioteca.clacso.edu.ar/clacso/ se/20200515065052/28-Julio-C-Gambina.pdf

GEORGIEVA, Kristalina. "Managing Director's Speaking Points at 2020 Spring Meetings IMFC Plenary". FMI, 16 de abril de 2020. Disponible en https://www.imf.org/en/News/Articles/2020/04/16/sp041620managing-director-speaking-points-at-imfc

HARMAN, Sophie. "Ébola, género y mujeres notablemente invisibles en la gobernanza de la salud mundial". Third World Quaterly, v. 37, n. 3, 2015.

INSTITUTO DE PESQUISA ECONOMICA APLICADA (IPEA). Retrato das desigualdades de gênero e raça (Brasil, 2015). Disponible en: https://www.ipea.gov.br/retrato/apresentacao.html

KENTIKELENIS, Alexander et al.. "Softening the blow of the pandemic: will the International Monetary Fund and World Bank make things worse?". Lancet Glob Health, 9 de abril de 2020. Disponible en https://www.thelancet.com/action/showPdf?pii=S2214-109X\%2820\%2930135-2

KENTIKELENIS, Alexander et al.. "IMF conditionality and development policy space, 1985-2014". Review of International Political Economy, v. 23, n. 4, 2016.

KOOB, Sigrid; JORGENSEN, Stinne; SANO, Hans-Otto Sano. Human Rights and Economic Growth: An Econometric Analysis of Freedom and Participant. Denmark: Danish Institute for Human Rights, Matters of Concern - Human Rights' Research Papers, n. 4, 2018.

LANAU, Sergi; FORTUN, Jonathan. "The Covid-19 Shock to EM Flows". Economic Views, Institute of International Finance, 17 de marzo de 2020. Disponible en, https://www.iif.com/Publications/ID/ 3802/Economic-Views-The-COVID-19-Shock-to-EM-Flows

MALPASS, David. "Remarks by World Bank Group President on G20 Finance Ministers Conference Call on COVID-19". 23 de marzo de 2020. Disponible en https://www.worldbank.org/en/news/speech/ 
2020/03/23/remarks-by-world-bank-group-president-david-malpass-on-g20-finance-ministersconference-call-on-covid-19

NACIONES UNIDAS. "Covid-19 and human rights. We are all in this together". abril de 2020a, Nueva York. Disponible en https://www.un.org/sites/un2.un.org/files/un_policy_brief_on_human_rights_and_ covid_23_april_2020.pdf

NACIONES UNIDAS. "Policy Brief: The Impact of COVID-19 on Women". 9 de abril 2020b. Disponible en https://www.unwomen.org/en/digital-library/publications/2020/04/policy-brief-the-impact-ofcovid-19-on-women

NACIONES UNIDAS. "Funding mechanisms for COVID-19 response". Departamento de Asuntos Económicos y Sociales de la ONU, abril de 2020c. Disponible en https://www.un.org/development/ desa/financing/sites/www.un.org.development.desa.financing/files/2020-05/Mapping\%20of\%20CO VID-19\%20Response.pdf

OIT. Políticas de formalización del trabajo doméstico remunerado en América Latina y el Caribe. Programa de Promoción de la Formalización en América Latina y el Caribe (FORLAC), Lima, 2016.

ONU Mujeres. "COVID-19 en América Latina y el Caribe: Cómo incorporar a las mujeres y la igualdad de género en la gestión de la respuesta de la crisis". Informe de ONU Mujeres, marzo 2020. Disponible en: https://lac.unwomen.org/es/digiteca/publicaciones/2020/03/covid-como-incorporara-las-mujeres-y-la-igualdad-de-genero-en-la-gestion-de-respuesta

OPS-ORGANIZACIÓN PANAMERICANA DE LA SALUD. "Actualización epidemiológica: dengue". Informe de la OPS, 7 de febrero de 2020. Disponible en: https://www.paho.org/sites/default/files/2020-02/ 2020-feb-7-phe-actualizacion-epi-dengue.pdf

PIRAS, Claudia. Las mujeres en América Latina y el caribe enfrentan mayores riesgos ante el coronavirus. Blog, 23 de marzo de 2020. Disponible en: https://blogs.iadb.org/igualdad/es/mujeresenfrentan-mayores-riesgos-ante-coronavirus/

QUIJANO, Aníbal. “Colonialidad del Poder, Eurocentrismo y América Latina”. En LANDER, E. (Comp.). La colonialidad del saber: eurocentrismo y ciencias sociales. Perspectivas Latinoamericanas. ${ }^{a}$ ed. Buenos Aires: Fundación Centro de Integración, Comunicación, Cultura y Sociedad -CICCUS; Consejo Latinoamericano de Ciencias Sociales -CLACSO, 2011.

QUIJANO, Aníbal. "Colonialidade, poder, globalização e democracia”. Novos Rumos, São Paulo ano 17, n. 37, p. 4-29, Instituto Astrojildo Pereyra, 2002.

RAZAVI, Shahra. The Political and Social Economy of Care in a Development Context: Conceptual Issues, Research Questions and Policy Options. Ginebra: Gender and Development - Programme Paper Nuìm. 3, Instituto de Investigaciones de las Naciones Unidas para el Desarrollo Social, June 2007.

REDIKER, Douglas; CREBO-REDIKER, Heidi. "COVID-19 uncertainty and the IMF". Blog, Brookings Institution, mayo de 2020. Disponible en https://www.brookings.edu/blog/future-development/2020/ 04/14/covid-19-uncertainty-and-the-imf/

RODRÍGUEZ ENRÍQUEZ, Corina. "Economía feminista y economía del cuidado. Aportes conceptuales para el estudio de la desigualdad”. Nueva Sociedad, Buenos Aires, n. 256, marzo -abril 2015.

RODRÍGUEZ ENRÍQUEZ, Corina; PAUTASSI, Laura. “Violencia contra las mujeres y políticas públicas. Implicancias fiscales y socioeconómicas". CEPAL, Serie Asuntos de Género, n. 137, 2016.

ROWDEN, Rick. From the Washington Consensus to the Wall Street Consensus. The financialization initiative of the World Bank and multilateral development Banks. Washington, DC: Heinrich Boll Stiftung, octubre de 2019. Disponible en https://us.boell.org/2019/10/11/washington-consensuswall-street-consensus

ROMERO, María José et. al.. "América Latina y la epidemia de COVID-10: una crónica de múltiples crisis (I)". European network on debt and development (EURODAD), 30 de marzo de 2020. Disponible en: https://www.latindadd.org/2020/03/30/america-latina-y-la-epidemia-de-covid-19-una-cronicade-multiples-crisis-i/

RUCKERT, Arne; LABONTÉ, Ronald. "Public-private partnerships (PPPs) in global health: the good, the bad and the ugly". Third World Quarterly, n. 35, p. 1598-614, 2014. 
SKIDELSKY, Robert; FRACCAROLI, Nicolo. Austerity vs. Stimulus: The Political Future of Economic Recovery. Basingstoke: Palgrave Macmillan, 2017.

STEIL, Benn. La batalla de Bretton Woods. John Maynard Keynes, Harry Dexter White y cómo se fraguó un nuevo orden mundial. Barcelona: Deusto, 2016.

UNICEF. "COVID-19: Más del 95 por ciento de niños, niñas y adolescentes está fuera de las escuelas en América Latina y el Caribe". Informe UNICEF, 23 de marzo de 2020. Disponible en https:// www.unicef.org/lac/comunicados-prensa/covid-19-mas-del-95-por-ciento-de-ninos-y-niñas-estafuera-de-las-escuelas

VÁSCONEZ RODRÍGUEZ, Alison. "Crecimiento econoìmico y desigualdad de geìnero: anailisis de panel para cinco paiises de Ameìica Latina". Revista CEPAL, n. 122, 2017. Disponible en https:// repositorio.cepal.org/bitstream/handle/1 1362/42033/1/RVE122_Vasconez.pdf

Juan Pablo Bohoslavsky (juanpablobohos@gmail.com) fue entre 2014 y 2020 Experto Independiente en Deuda Externa y Derechos Humanos de la ONU. Trabajó para la Unctad y fue consultor de empresas del Estado argentino y de la Cepal. Doctor en Derecho. Su última publicación es "Austerity measures and women's social and economic rights: We need to look deeper", en Binder et al. (eds.), Research Handbook on International Law and Social Rights, Edward Elgar Publishing, 2020 (en prensa, en coautoría con Abby Kendrick).

Mariana Rulli (mrulli@unrn.edu.ar) es politóloga (UBA) y doctora en Ciencias Sociales de la FLACSO. Es docente e investigadora de la Universidad Nacional de Río Negro. Su último artículo es "Madres y/o Políticas: entre el derecho a participar y el derecho al cuidado" en Laura Pautassi (ed.), Géneros, Justicia y Políticas Públicas, Colección Género, Derecho y Justicia dirigida por Marisa Herrera, Natalia de la Torre y Silvia Fernández, Editorial Rubinzal Culzoni: Buenos Aires.

\section{COMO CITAR ESTE ARTÍCULO, DE ACUERDO CON LAS NORMAS DE LA REVISTA:}

BOHOSLAVSKY, Juan Pablo; RULLI, Mariana. "Covid-19, instituciones financieras internacionales y continuidad de las políticas androcéntricas en América Latina". Revista Estudios Feministas, Florianópolis, v. 28, n. 2, e73510, 2020.

\section{CONTRIBUCIÓN DE AUTORÍA}

Coautoría. Juan Pablo Bohoslavsky: concepción, recolección de datos y análisis de datos, elaboración del manuscrito, redacción, discusión de resultados.

Mariana Rulli: concepción, recolección de datos y análisis de datos, elaboración del manuscrito, redacción, discusión de resultados.

\section{FINANCIACIÓN}

No se aplica.

\section{CONSENTIMIENTO DE USO DE IMAGEN}

No se aplica.

APROBACIÓN DE COMITÉ DE ÉTICA EN INVESTIGACIÓN

No se aplica.

\section{CONFLICTO DE INTERESES}

No se aplica.

LICENCIA DE USO

Este artículo está licenciado bajo la Licencia Creative Commons CC-BY Internacional. Con esta licencia se puede compartir, adaptar, crear material para cualquier objetivo, siempre que se le atribuya la autoría.

HISTORIAL

Recibido el 06/05/2020

Presentado nuevamente el 22/05/2020

Aprobado el 29/05/2020 